\title{
Compósitos cimentícios leves reforçados com partículas de ma- deira de Eucalyptus grandis
}

\author{
Monica Regina Garcez ${ }^{1 \star}$, Estela Oliari Garcez ${ }^{2}$, Aline de Oliveira Machado ${ }^{3}$, Darci Alberto Gatto ${ }^{3}$ \\ ${ }^{1}$ Universidade Federal do Rio Grande do Sul, Tramandaí, Brasil. \\ ${ }^{2}$ Deakin University, Geelog, Austrália. \\ ${ }^{3}$ Centro de Engenharias, Universidade Federal de Pelotas, Pelotas, Brasil.
}

\begin{abstract}
RESUMO O objetivo principal deste trabalho foi a caracterização de um compósito cimentício de baixa densidade reforçado com partículas de madeira de Eucalyptus grandis, por meio do estudo de suas propriedades físicas e mecânicas. O traço padrão foi definido a partir de compósitos com $30 \%$ de cimento e $70 \%$ de madeira, em volume, com diferentes quantidades de água e aditivo superplastificante. Foram testadas diferentes granulometrias para a de serragem de Eucalyptus grandis com o intuito de elevar a resistência à tração do compósito. O superplastificante usado com função de aumentar a fluidez da pasta proporcionou aumento de até $161,8 \%$ na resistência à compressão e de até 205,9\% e 123,46\% nos módulos de elasticidade estático e dinâmico dos compósitos. A resistência à tração dos compósitos foi em média 55,8\% superior quando foram utilizadas partículas de serragem retidas na peneira $2,36 \mathrm{~mm}$.
\end{abstract}

Palavras-chave: resistência à tração; propriedades físicas e mecânicas; serragem.

\section{Lightweight cementitious composites reinforced with Eucalyptus grandis wood particles}

\begin{abstract}
The aim of this research was to characterize a lightweight cementitious composite reinforced with Eucalyptus grandis wood particles through the study of its physical and mechanical properties. Standard mix proportion was defined based on a composite with $30 \%$ cement and $70 \%$ wood, in volume, and different amounts of water and superplasticizer. Different particle sizes of Eucalyptus grandis sawdust were tested to increase the composite tensile strength. The superplasticizer used with the function of enhancing paste fluidity provided an increase up to $161.6 \%$ in the compressive strength and up to $205.9 \%$ and $123.46 \%$ in the static and dynamic modulus of elasticity of the composites. Tensile strength presented was $55.8 \%$ higher when sawdust particles retained on the $2.36 \mathrm{~mm}$ sieve were used.
\end{abstract}

Keywords: tensile strength; physical and mechanical properties; sawdust.

\section{Introdução}

As indústrias de base florestal que atuam na transformação primária, como as serrarias, em geral apresentam baixo rendimento e geram grande quantidade de resíduos, que podem provocar problemas nas culturas agrícolas e florestais, quando seus extrativos atingem níveis tóxicos para as plantas, devido ao tempo necessário para sua degradação natural e ao grande volume muitas vezes depositado no mesmo local. Como muitas vezes estão localizadas em locais afastados dos grandes centros consumidores, a disposição inadequada dos resíduos acaba por gerar grandes passivos ambientais, devido principalmente ao alto custo de transporte (BARBOSA et al., 2014; RONQUIM et al., 2014).

Estudos recentes sobre a utilização de resíduos de madeira, na forma de fibras ou partículas, vem demonstrando o seu potencial para uso como reforço ou material de preenchimento em compósitos cimentícios (ASHORI et al., 2012; TORKAMAN et al., 2014; BERTOLINI et al., 2014; FAN et al., 2012; LIN et al., 1994; PEHANICHA, et al., 2004). Esses compósitos em geral apresentam baixa massa específica, 
baixa condutividade térmica, resistência ao fogo, impermeabilidade e resistência mecânica adequada para aplicação em placas delgadas, blocos para alvenaria e elementos de enchimentos para lajes, de uso frequente na construção civil (PIMENTEL et al., 2006).

O consumo de madeira serrada no Brasil atingiu 9,2 milhões de $\mathrm{m}^{3}$ em 2012 (ABRAF, 2013). O rendimento do $E$ ucalyptus grandis pode variar de $40 \%$ a $60 \%$, dependendo da técnica de desdobro utilizada (CUNHA, 2015). Desta forma, considerando a disponibilidade do resíduo e o fato de serem consideradas moderadamente inibitórias (BERALDO e CARVALHO, 2004; MORI et al., 2007) ou até mesmo naturalmente compatíveis com o cimento (CASTRO et al., 2014), não requerendo tratamento superficial prévio para evitar problemas de compatibilidade, a utilização de serragem de Eucalyptus grandis na produção de compósitos cimento-madeira torna-se interessante e vantajosa.

Neste contexto, este trabalho tem como objetivo a caracterização de um traço padrão para um compósito cimentício de baixa densidade, reforçado com partículas de madeira de Eucalyptus grandis, com $30 \%$ de cimento e $70 \%$ de madeira, em volume, por meio do estudo de suas propriedades físicas e mecânicas, buscando também elevar a resistência à tração dos compósitos através da variação na granulometria das partículas de madeira.

\section{Material e Métodos}

\section{Materiais}

Os compósitos foram fabricados com serragem de $\mathrm{Eu}$ calyptus grandis $\left(\rho_{\mathrm{un}}=0,12 \mathrm{~g} / \mathrm{cm}^{3}\right)$, in natura, material passante na peneira 2,36mm, e cimento CP V-ARI RS, cujas especificações atendem a NBR 5733 (ABNT, 1991). Em alguns traços foi utilizado aditivo superplastificante Sika ViscoCrete ${ }^{\varpi}$
5700, cuja composição básica consiste em uma solução de policarboxilato em meio aquoso, que atende aos requisitos da NBR 11768 (ABNT, 2011).

\section{Delineamento experimental}

A partir de um traço padrão 1:0,28:0,6 (cimento:madeira:água, em massa), que corresponde à proporção 30:70 (cimento:madeira, em volume), foram determinadas as proporções mostradas na Tabela 1. Buscando obter misturas mais trabalháveis, com adequada coesão e fácil moldagem, que possibilitassem aumento na resistência à compressão, foi testada a utilização de aditivo superplastificante nos compósitos II, III e IV, mantendo o traço padrão (I).

Tabela 1. Proporção dos materiais.

Table 1. Proportion of the materials

\begin{tabular}{cccc}
\hline & $\begin{array}{c}\text { cimento:madeira } \\
\end{array}$ & $\begin{array}{c}\text { água:cimento } \\
\star \star\end{array}$ & $\begin{array}{c}\text { aditivo:cimento } \\
\star \star\end{array}$ \\
\hline I & $30: 70$ & 0,6 & - \\
II & $30: 70$ & 0,6 & 0,005 \\
III & $30: 70$ & 0,6 & 0,006 \\
IV & $30: 70$ & 0,6 & 0,007 \\
V & $30: 70$ & 0,5 & 0,008 \\
VI & $30: 70$ & 0,7 & - \\
\hline
\end{tabular}

*em volume; ${ }^{\star *}$ em massa, máxima dosagem 0,015 segundo as recomendações do fabricante.

\section{Produção dos compósitos}

Os materiais foram misturados em uma argamassadeira e os compósitos foram moldados em cilindros de 50mm de diâmetro e 100mm de altura, conforme a NBR 7215 (ABNT, 1996). Após a moldagem, foram mantidas placas de vidro quadradas $(70 \times 70 \mathrm{~mm})$ sobre os moldes por $24 \mathrm{~h}$, para a cura inicial dos compósitos. Decorridas $24 \mathrm{~h}$ da moldagem, foi realizada a desforma e os corpos de prova foram então mantidos submersos em um tanque de cura saturado de cal, conforme NBR 9479 (ABNT, 1984), até atingirem a idade requerida para os ensaios. 


\section{Avaliação das propriedade físicas e mecânicas}

\section{dos compósitos}

Para analisar a influência das diferentes proporções de materiais nas propriedades físicas e mecânicas dos compósitos, foram determinadas as massas específicas aparentes com teor de umidade estabilizado em $12 \%$ e foram realizados ensaios de resistência à compressão, segundo a NBR 7215 (ABNT,1997), e módulo de elasticidade estático, segundo a NBR 8522 (ABNT, 2003). O módulo de elasticidade dinâmico dos compósitos foi determinado por meio de medições de velocidade de pulso ultrassônico, com o aparelho de Ultrassom da marca Proceq, modelo Tico e transdutores de $54 \mathrm{kHz}$, segundo a NBR 15630 (ABNT, 2008). Os ensaios foram realizados em 4 amostras de cada compósito, em corpos de prova cilíndricos de $5 \mathrm{~cm}$ de diâmetro e $10 \mathrm{~cm}$ de altura, na idade de 28 dias.

Para o traço com melhores propriedades mecânicas e possibilidade de utilização da menor quantidade de aditivo possível, por questões ambientais e econômicas, foram determinados índice de vazios e absorção de água, em 4 corpos de prova cilíndricos de $5 \mathrm{~cm}$ de diâmetro e $10 \mathrm{~cm}$ de altura, na idade de 28 dias, segundo a NBR 9778 (ABNT, 2005). A partir deste traço foi analisada a resistência à tração na flexão do compósito, segundo os procedimentos da NBR 13279 (ABNT, 2005), e foram determinadas mais duas variações de granulometria para as partículas de serragem de Eucalyptus grandis (Tabela 2), in natura, visando alternativas para elevar a tração na flexão de compósitos cimento-madeira. Os ensaios de flexão estática a 3 pontos foram realizados em 3 amostras de corpos-de-prova prismáticos, com dimensões $40 \mathrm{~mm} \times 40 \mathrm{~mm} \times 160 \mathrm{~mm}$, aos 28 dias.
Tabela 2. Proporções dos materiais para o ensaio de flexão a 3 pontos.

Table 2. Proportions of the materials for the 3-point static bending test.

\begin{tabular}{cccc}
\hline & $\begin{array}{c}\text { cimento:madeira } \\
\end{array}$ & $\begin{array}{c}\text { água:cimento } \\
* *\end{array}$ & $\begin{array}{c}\text { Granulometria } \\
\text { madeira }\end{array}$ \\
\hline $\mathrm{VI}$ & $30: 70$ & 0,7 & $100: 0$ \\
$\mathrm{VIa}$ & $30: 70$ & 0,7 & $80: 20$ \\
$\mathrm{VIb}$ & $30: 70$ & 0,7 & $50: 50$ \\
\hline
\end{tabular}

${ }^{\star}$ em volume; ${ }^{\star *}$ em massa; ${ }^{* *}$ em porcentagem, passante:retido na peneira $2,36 \mathrm{~mm}$.

\section{Análise estatística}

Foi realizada análise de variância (ANOVA) utilizando o software Statgraphics. Para discriminar a diferença entre médias foi utilizado o teste da diferença mínima significativa (LSD) de Fischer, em nivel de 5\% de probabilidade de erro.

\section{Resultados e Discussão}

Os valores de resistência à compressão, módulo de elasticidade e massa específica obtidos dos compósitos (Tabela 3) permitem que estes sejam utilizados em certas aplicações práticas da construção civil (BOUGUERRA, 1998), como, por exemplo, a fabricação de blocos de vedação e artefatos de cimento.

O compósito VI, com traço 1:0,28:0,7 sem utilização de aditivo superplastificante apresentou resistência à compressão compatível com as obtidas nos trabalhos de Rodrigues et al. (2007), 12,5MPa (traço 1:0,37:0,7) e Beraldo e Carvalho (2004), 8,4MPa (traço 1:0,375:0,75), fabricados com cimento ARI, resíduo de madeira de Eucaliptus grandi com diâmetro máximo 2,36mm e traços cimento:madeira:água expressos em massa.

A utilização do aditivo superplastificante aumentou a resistência à compressão dos compósitos II, III e IV em relação ao compósito I em $62,68 \%, 58,38 \%$ e $161,8 \%$, respectivamente (Tabela 3). O mesmo ocorreu com os módulos de elasticidade estático $(50 \%, 31,81 \%$ e $205,9 \%)$ e dinâmico $(35,71 \%, 16,66 \%$ 
e 123,46\%). O uso do aditivo superplastificante, portanto, melhorou o desempenho dos compósitos com relação às suas propriedades mecânicas.

Em geral, maiores valores de massa específica estão associados a maiores valores de módulo de elasticidade (IWAKIRI et al.,2008) e limites de resistência, como comprovam os dados da Tabela 3 e a Figura 1. Massas específicas, resistências à compressão e módulos de elasticidade mais elevados são características de compósitos mais rígidos, por outro lado, em alguns casos, o uso de peças mais leves, com menor massa específica, podem ser uma vantagem em relação a outros quesitos, como transporte manuseio e montagem.

Tabela 3. Resultados experimentais das propriedades físicas e mecânicas.

Table 3. Experimental results of the physical and the mechanical properties.

\begin{tabular}{|c|c|c|c|c|c|c|c|c|c|c|c|c|}
\hline & \multicolumn{2}{|c|}{$\begin{array}{l}\text { Massa específica } \\
\text { aparente }\left(\mathrm{g} / \mathrm{cm}^{3}\right)\end{array}$} & \multicolumn{2}{|c|}{$\begin{array}{l}\text { Resistência à } \\
\text { compressão } \\
(\mathrm{MPa})\end{array}$} & \multicolumn{2}{|c|}{$\begin{array}{c}\text { Módulo de elastici- } \\
\text { dade estático } \\
(\mathrm{GPa})\end{array}$} & \multicolumn{2}{|c|}{$\begin{array}{l}\text { Módulo de elastici- } \\
\text { dade dinâmico } \\
(\mathrm{GPa})\end{array}$} & \multicolumn{2}{|c|}{$\begin{array}{l}\text { Absorção de } \\
\text { água } \\
(\%)\end{array}$} & \multicolumn{2}{|c|}{$\begin{array}{l}\text { Índice de vazios } \\
\qquad(\%)\end{array}$} \\
\hline & Média & CV (\%) & Média & CV (\%) & Média & CV (\%) & Média & CV (\%) & Média & CV (\%) & Média & CV (\%) \\
\hline I & $1,27^{\mathrm{a}}$ & 3,02 & $7,45^{a}$ & 4,37 & $2,20^{a}$ & 14,37 & $2,94^{\mathrm{a}}$ & 5,71 & - & - & - & - \\
\hline II & $1,35^{\mathrm{b}}$ & 1,53 & $12,12^{b}$ & 2,40 & $3,30^{\mathrm{b}}$ & 11,87 & $3,99^{\mathrm{b}}$ & 8,39 & - & - & - & - \\
\hline III & $1,29^{\mathrm{ac}}$ & 1,62 & $11,88^{b}$ & 5,52 & $2,90^{\mathrm{b}}$ & 18,46 & $3,43^{c}$ & 7,46 & - & - & - & - \\
\hline IV & $1,46^{\mathrm{d}}$ & 5,48 & $19,51^{\mathrm{c}}$ & 0,96 & $6,73^{c}$ & 1,71 & $6,57^{\mathrm{d}}$ & 1,71 & - & - & - & - \\
\hline $\mathrm{V}$ & $1,21^{\mathrm{e}}$ & 2,78 & $7,34^{\mathrm{a}}$ & 6,05 & $0,97^{\mathrm{d}}$ & 26,03 & $2,11^{\mathrm{e}}$ & 12,71 & - & - & - & - \\
\hline VI & $1,33^{b c}$ & 0,75 & $14,04^{\mathrm{d}}$ & 3,81 & $4,50^{\mathrm{e}}$ & 8,01 & $4,18^{\mathrm{b}}$ & 5,40 & 38,60 & 4,82 & 39,31 & 4,90 \\
\hline
\end{tabular}

Médias seguidas pela mesma letra dentro da mesma coluna não diferenciam estatisticamente, a $5 \%$ de probabilidade de erro, pelo teste LSD de Fischer; CV = coeficiente de variação.

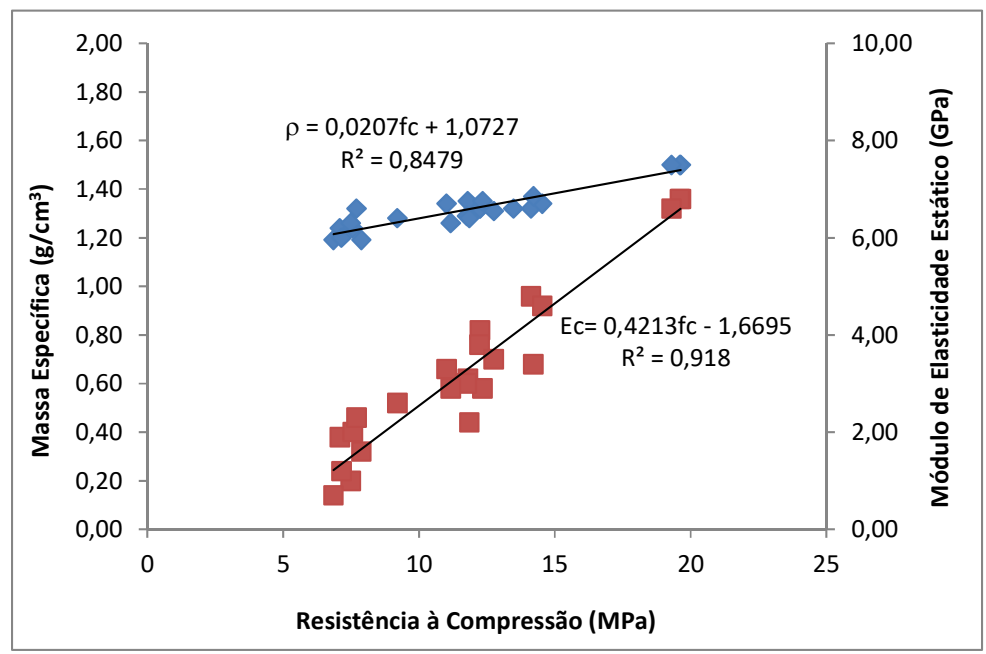

Figura 1. Relação entre resistência à compressão, massa específica aparente e módulo de elasticidade estático.

Figure 1. Relationship between compressive strength, density and static modulus of elasticity.

Devido às características higroscópicas da madeira, a diminuição da quantidade de água não foi efetiva para aumentar a resistência à compressão do traço V (Tabela 3), que mostrou também redução nos módulos de elasticidade. Isto significa que a quantidade de água disponível não foi suficiente para hidratar as partículas de cimento e que o aditivo beneficiou o comportamento mecânico do compósito somente quando foi utilizado com a função de aumentar a fluidez da pasta. Adicionalmente, a resistência à compressão dos traços V e I não variou estatisticamente e, embora o traço VI apresente $10 \%$ a mais de água que os traços I (sem aditivo), II e III 
(com aditivo), este resultou maior resistência à compressão $(88,45 \%, 15,84 \%$ e $18,18 \%)$ e módulo de elasticidade estático (104,54\%, 36,36\%, 55,17\%) em relação a estes compósitos.

O compósito VI apresentou absorção de água e índice de vazios compatíveis com os de compósitos com mesma espécie e similares percentuais de madeira encontrados na literatura (ARSÉNE et al., 2003; DEL MENEZZI et al., 2000), que variam de $14 \%$ a $40 \%$ dependendo da composição do traço, quantidade de água e utilização ou não de adições minerais e aditivos. Valores desta magnitude, 38,60\% de absorção de água e 39,31\% de índice de vazios para uma proporção cimento:madeira:água de 1:0,28:0,7 em massa ocorrem devido a característica higroscópica da madeira, que aliada à porosidade da matriz, permite o preenchimento dos vazios existentes pela água (TORKAMAN et al., 2014).

A velocidade propagação da onda ultrassônica, além de permitir a determinação do módulo de elasticidade dinâmico dos compósitos, pode ser utilizada como ferramenta auxiliar para estimar o módulo de elasticidade estático de compósitos cimento-madeira (Figura 2), o que pode ser comprovado pelo ajustamento do modelo estatístico $(91,48 \%)$.

Tabela 4. Resultados dos ensaios de flexão a 3 pontos. Table 4. Results of 3-point static bending tests.

\begin{tabular}{ccc}
\hline & \multicolumn{2}{c}{ Resistência à tração na flexão (MPa) } \\
\hline & Média & CV (\%) \\
VI & $5,42^{\mathrm{a}}$ & 16,46 \\
$\mathrm{VIa}$ & $8,54^{\mathrm{b}}$ & 11,94 \\
$\mathrm{VIb}$ & $8,35^{\mathrm{b}}$ & 7,33 \\
\hline
\end{tabular}

Médias seguidas pela mesma letra dentro da mesma coluna não diferenciam estatisticamente, a $5 \%$ de probabilidade de erro, pelo teste LSD de Fischer; CV = coeficiente de variação.

A resistência à tração na flexão dos compósitos (Tabela 4) aumentou significantemente nos traços VIa (57,56\%) e VIb (54,05\%), em relação ao VI, cujos aspectos visuais são mostrados na Figura 3.

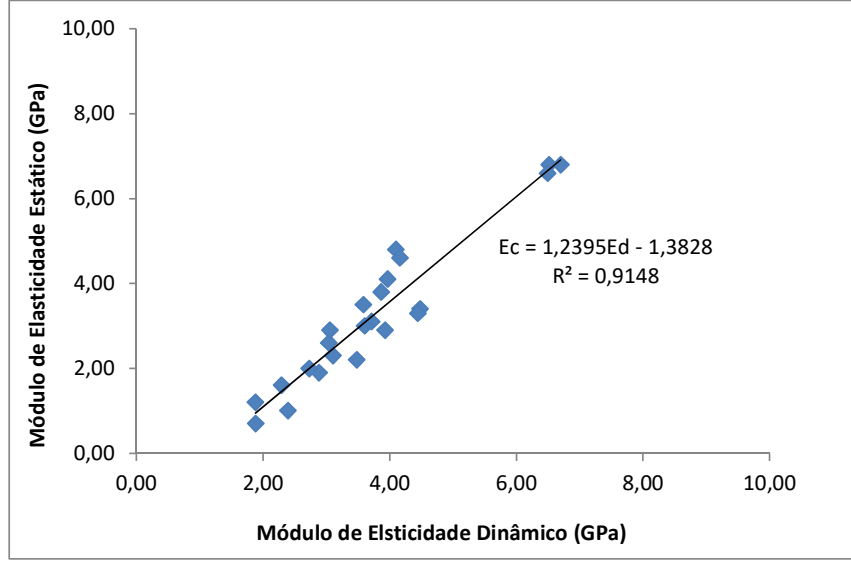

Figura 2. Relação entre módulos de elasticidade estático e dinâmico.

Figure 2. Relationship between static and dynamic modulus of elasticity.

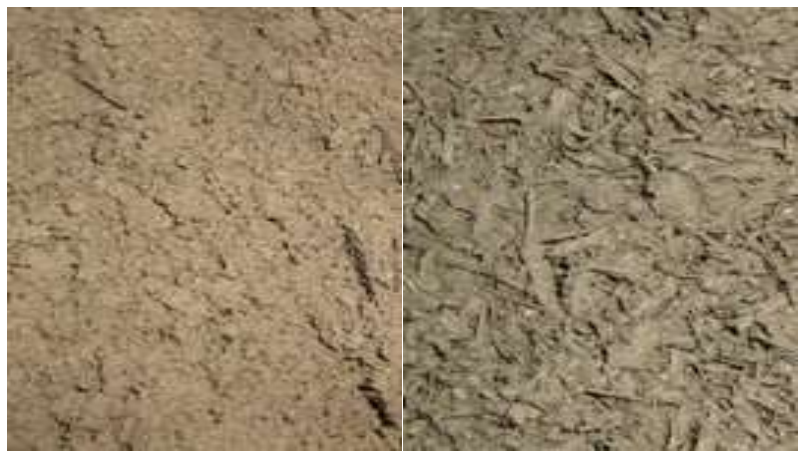

Figura 3. Aspecto visual das misturas dos traços VI (esquerda) e VIb (direita).

Figure 3. Visual aspect of mix proportions VI (left) and VIb (right).

A resistência à tração do compósito cimento-madeira VI (Tabela 4) corresponde a 38,60\% de sua resistência à compressão (Tabela 3). Em compósitos cimentícios convencionais, do tipo concreto, sem adição de fibras, resistência à tração equivale a 10\% da resistência à compressão, enquanto em argamassas equivale a $6 \%$, dependendo do proporcionamento de água, agregados e cimento na pasta (STILL e THOMAS, 2006). As particulas maiores de serragem, presentes nos traços VIa e VIb, portanto, auxiliaram na transferência de tensões quando solicitadas ao carregamento, proporcionando aos compósitos VIa e VIb maior capacidade de deformação em relação ao VI (Figura 3). Entretanto, não houve diferença estatística entre os valores de resistência tração dos compósitos VIa e VIb, sinalizando a necessidade de um 
proporcionamento adequado com referência ao tamanho das partículas.

\section{Conclusões}

O traço para compósito cimentício leve desenvolvido neste trabalho, com $30 \%$ de cimento ARI-RS e $70 \%$ de serragem de Eucalyptus grandis, apresentou resultados propriedades físicas e mecânicas compatíveis com aplicações práticas.

O uso do aditivo superplastificante melhorou o desempenho dos compósitos com relação às suas propriedades mecânicas, quando foi utilizado com a função de aumentar a fluidez da pasta, uma vez que a quantidade de água disponível na pasta deve ser suficiente para hidratar as partículas de cimento.

A resistência à tração na flexão dos compósitos aumentou de forma significativa nos traços em que foram utilizadas partículas de serragem com material retido na peneira $2,36 \mathrm{~mm}$. As fibras de madeira permitiram a transferência das tensões da matriz de cimento proporcionando aos compósitos maior capacidade de deformação.

A realização do ensaio não destrutivo, por meio do aparelho de ultrassom com transdutores de face plana pode ser utilizada como ferramenta auxiliar para estimar e relacionar as propriedades mecânicas dos compósitos cimento-madeira.

\section{Referências}

ARSĖNE M. A.; SAVASTANO JR, H.; ALLAMEH, S M.; GHAVAMI, K; SOBOYEJO W. Cementitious composites reinforced with vegetable fibers. First Interamerican Conference on Non-Conventional Materials and Technologies in the Eco-Construction and Infrastructure, 2003, João Pessoa. Proceedings... João Pessoa, 2003.

ASHORI, A.; TABARSA, T.; AMOSI, F. Evaluation of using waste timber railway sleepers in wood-cement composite materials. Construction and Building Materials, n. 27, p. 126-129, 2012.
ASSOCIAÇÃO BRASILEIRA DE NORMAS TÉCNICAS. NBR 5733: Cimento Portland de alta resistência inicial. Rio de Janeiro, 1991.

ASSOCIAÇÃO BRASILEIRA DE NORMAS TÉCNICAS. NBR 7215: Cimento Portland - Determinação da resistência à compressão. Rio de Janeiro, 1997.

ASSOCIAÇÃO BRASILEIRA DE NORMAS TÉCNICAS. NBR 8522: Concreto - Determinação dos módulos estáticos de elasticidade e de deformação e da curva tensão-deformação. Rio Janeiro, 2003.

ASSOCIAÇÃO BRASILEIRA DE NORMAS TÉCNICAS (ABNT). NBR 9479: Câmaras úmidas e tanques para cura de corpos-de-prova de argamassa e concreto. Rio de Janeiro: ABNT, 1984. 2p.

ASSOCIAÇÃO BRASILEIRA DE NORMAS TÉCNICAS. NBR 9778: Argamassa e concreto endurecidos - Determinação da absorção de água, índice de vazios e massa específica - Requisitos. Rio de Janeiro, 2005.

ASSOCIAÇÃO BRASILEIRA DE NORMAS TÉCNICAS. NBR 11768: Aditivos químicos para concreto de cimento Portland - Requisitos. Rio de Janeiro, 2011.

ASSOCIAÇÃO BRASILEIRA DE NORMAS TÉCNICAS. NBR 13279: Argamassa para assentamento e revestimento de paredes e tetos - Determinação da resistência à tração na flexão e à compressão. Rio de Janeiro, 2005.

ASSOCIAÇÃO BRASILEIRA DE NORMAS TÉCNICAS. NBR 15630: Argamassa para assentamento e revestimento de paredes e tetos - Determinação do módulo de elasticidade dinâmico através da propagação de onda ultra-sônica. Rio de Janeiro, 2008.

ASSOCIAÇÃO BRASILEIRA DE PRODUTORES DE FLORESTAS PLANTADAS. Anuário estatístico da ABRAF 2013 ano base 2012. Brasília, 2013, 146p.

BARBOSA, L. C., PEDRAZZI, C., FERREIRA, E. S., SCHNEID, G. N., WILLE, V. K. D. Avaliação de resíduos de uma serraria para produção de celulose kraft. Ciência Florestal, v. 24, n. 2, p. 491-500, 2014.

BERALDO, A.L.; CARVALHO J.V. Compósito Eucalyptus grandis - cimento Portland. Scientia Forestalis, n. 65, p.150161,2004 
BERTOLINI, M. S.; CAMPOS, C. I.; SOUZA, A. M.; PANZERA, T. H.; CHRISTOFORO, A. L.; LAHR, F. A. R. Woodcement composites from wastes of Pinus sp. wood: Effect of particles treatment. International Journal of Composite Materials, v. 4, n 2, p. 146-149, 2014.

BOUGUERRA, A.; LEDHEM, F.; BERQUIN, F.; DHEILLY, R.M.; QUÉNEUDEC, M. Effect of microstructure on the mechanical and thermal properties of lightweight concrete prepared from clay, cement, and wood aggregates. Cement and Concrete Research, v. 28, n.8, p. 1179-1190, 1998.

CASTRO V.; ARAÚJO R. D.; PARCHEN, C.; IWAKIRI, S. Avaliação dos efeitos de pré-tratamentos da madeira de $E$ u calyptus benthamii Maiden \& Cambage no grau de compatibilidade com cimento Portland. Revista Árvore, v. 38, n. 5, p. 945-952, 2014

CUNHA, A. B, FRANÇA, M. C., ALMEIDA, C. C. F., GORSKI, L., CRUZ, R. C., SANTOS, D. Avaliação do rendimento em madeira serrada de Eucalyptus benthamii e de Eucalyptus grandis por meio do desdobro tangencial e radial. Floresta, v. 45, n. 2, p. 241-250, 2015.

DEL MENEZZI, C. H. S.; SOUZA, M. R. Influence of bark on properties of wood-cement particleboards made with eucalypt. International Conference on Wood and Wood Fiber Composites, 2000, Stuttgart. Proceedings... Stuttgart, 2000.

FAN, M., NDIKONTAR, M. K., ZHOU, X., NGAMVENG, J. H. Cement-bonded composites made from tropical woods: Compatibility of Wood and cement. Construction and Building Materials, v. 36, p. 135-140, 2012.

IWAKIRI, S., STINGHEN, A. B. M., SILVEIRA, E. L., ZAMARIAN, E. H. C., PRATA, J. G., BRONOSKI, M. Influência da massa específica sobre as propriedades mecânicas de painéis aglomerados. Floresta, v. 38, n. 3, 2008.

LIN X., SILSBEE M. R., ROY D. M., KESSLER R., BLANKENHORN P. R. Approaches to improve the properties of wood fiber reinforced cementitious composites. Cement and Concrete Research, v. 24, n. 8, p. 1558-1566, 1994.

MORI, F. A.; LOPES, Y. L. V.; MENDES, L. M.; LATORRACA, J. V. F. estudo da compatibilidade entre a madeira e as cascas de Eucalyptus grandis e cimento Portland. Ciência Florestal, v. 17, n. 3, p. 257-264, 2007.
PEHANICHA J. L., BLANKENHORNA P. R., SILSBEEB M. R. Wood fiber surface treatment level effects on selected mechanical properties of wood fiber-cement composites. Cement and Concrete Research, n. 34, p. 59-65, 2004.

PIMENTEL, L. L.; BERALDO, A. L.; SAVASTANO JÚNIOR, H. Durabilidade de compósito biomassa vegetal-cimento modificado por polímero. Engenharia Agrícola, v.26, n.2, p.344353, 2006.

RODRIGUES, M. S.; FERREIRA, G. C.; VIEIRA, F. F.; BERALDO, A. L. Avaliação da compatibilidade química entre o cimento portland e resíduos vegetais por meio da velocidade do pulso ultra-sônico (VPU). Congresso Brasileiro de Engenharia Agrícola, 2007, Bonito. Proceedings... Bonito, 2007.

RONQUIM R. M., FERRO F. S., ICIMOTO F. H., CAMPOS C. I., BERTOLINI M. S., CHRISTOFORO A L., LAHR F. A. R. Physical and Mechanical Properties of Wood-Cement Composite with Lignocellulosic Grading Waste Variation. International Journal of Composite Materials, v. 4, n. 2, p. 69-72, 2014.

STILL, G.T.; THOMAS, T.H. Mix proportioning of mortars in tension, with particular reference to developing countries. Advances in Applied Ceramics, v. 105, n.4, p. 179-184, 2006.

TORKAMAN, J.; ASHORI, A.; MOMTAZI, A. S. Using wood fiber waste, rice husk ash, and limestone powder waste as cement replacement materials for lightweight concrete blocks. Construction and Building Materials, v.50, p. 432-436, 2014. 